Document downloaded from:

http://hdl.handle.net/10251/166263

This paper must be cited as:

Villamor, E.; Monserrat Aranda, C.; Del Río, L.; Romero-Martín, J.; Rupérez Moreno, MJ. (2020). Prediction of osteoporotic hip fracture in postmenopausal women through patientspecific FE analyses and machine learning. Computer Methods and Programs in Biomedicine. 193:1-11. https://doi.org/10.1016/j.cmpb.2020.105484

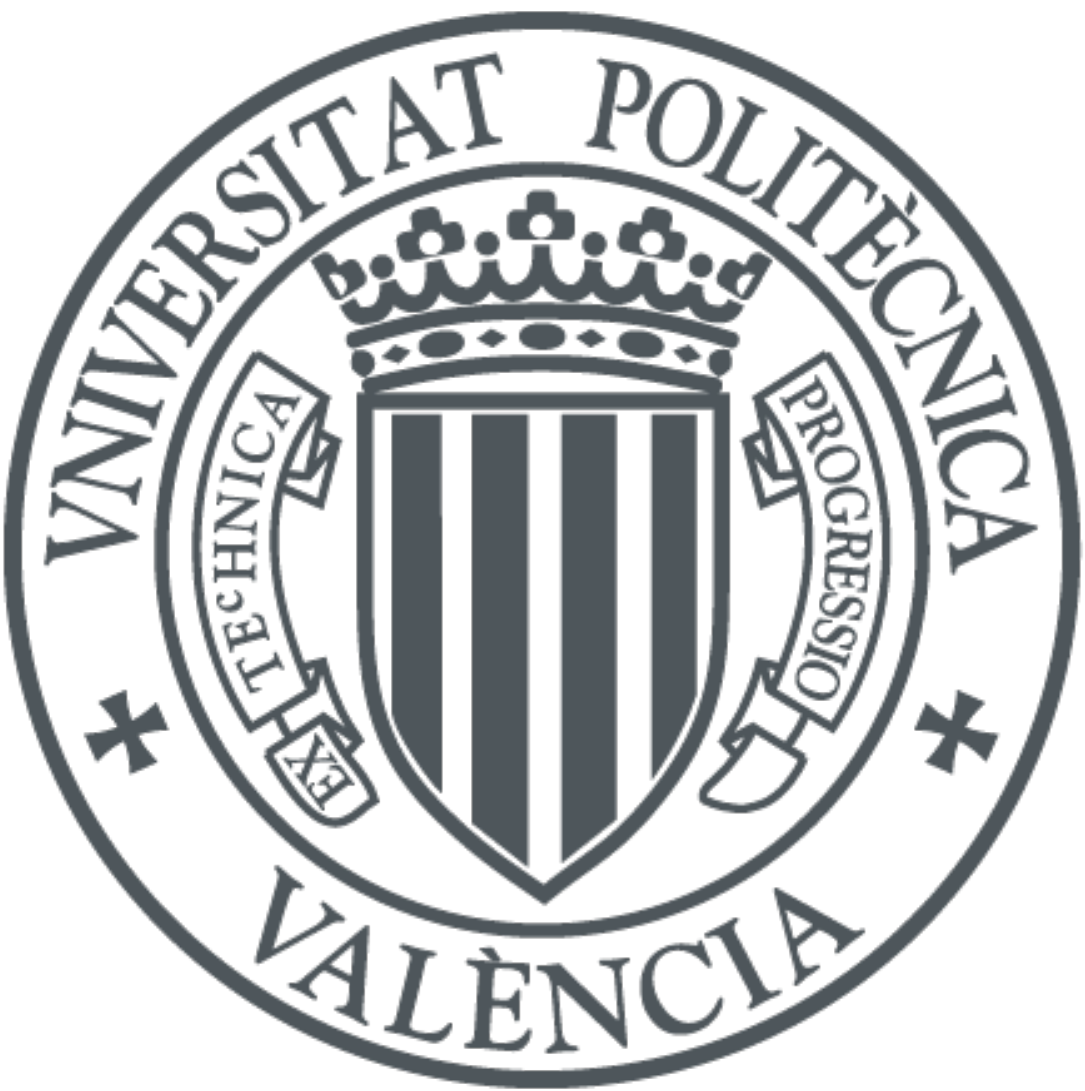

The final publication is available at

https://doi.org/10.1016/j.cmpb.2020.105484

Copyright Elsevier

Additional Information 


\title{
Prediction Of Osteoporotic Hip Fracture In Postmenopausal Women Through Patient-Specific FE Analyses And Machine Learning
}

\author{
E. Villamor ${ }^{\mathrm{a}}$, C. Monserrat ${ }^{\mathrm{a}}$, L. Del Ríob ${ }^{\mathrm{b}}$, J.A. Romero-Martín ${ }^{\mathrm{b}}$, M.J. \\ Rupérez ${ }^{\mathrm{c}, *}$ \\ ${ }^{a}$ Valencian Research Institute for Artificial Intelligence (VRAIN), Universitat Politècnica \\ de València, Camino de Vera $s / n, 46022$ Valencia, Spain \\ ${ }^{b}$ ASCIRES Grupo Biomédico, Valencia, Spain \\ ${ }^{c}$ Centro de Investigación en Ingeniería Mecánica, Universitat Politècnica de València, \\ Camino de Vera s/n, 46022 Valencia, Spain
}

\begin{abstract}
Background and objective: A great challenge in osteoporosis clinical assessment is identifying patients at higher risk of hip fracture. Bone Mineral Density (BMD) measured by Dual-Energy X-Ray Absorptiometry (DXA) is the current gold-standard, but its classification accuracy is limited to 65\%. DXA-based Finite Element (FE) models have been developed to predict the mechanical failure of the bone. Yet, their contribution has been modest. In this study, supervised machine learning (ML) is applied in conjunction with clinical and computationally driven mechanical attributes. Through this multi-technique approach, we aimed to obtain a predictive model that outperforms BMD and other clinical data alone, as well as to identify the best-learned ML classifier within a group of suitable algorithms.

Methods: A total number of 137 postmenopausal women $(81.4 \pm 6.95)$ were included in the study and separated into a fracture group $(n=89)$ and a control group $(n=48)$. A semi-automatic and patient-specific DXA-based FE model was used to generate mechanical attributes, describing the geometry, the impact force, bone structure and mechanical response of the bone after a sideways-fall. After preprocessing the whole dataset, 19 attributes were selected as predictors. Support Vector Machine (SVM) with radial basis function (RBF), Logistic Regression, Shallow Neural Networks and Random Forest were tested through a comprehensive validation procedure to compare their predictive performance. Clinical attributes were used alone in another experimental setup for the sake of comparison.

Results: SVM was confirmed to generate the best-learned algorithm for both experimental setups, including 19 attributes and only clinical attributes. The first, generated the best-learned model and outperformed BMD by $14 \mathrm{pp}$.

Conclusions: The results suggests that this approach could be easily integrated for effective prediction of hip fracture without interrupting the actual clinical workflow.
\end{abstract}

\footnotetext{
* Corresponding author at: Departamento de Ingeniería Mecánica y de Materiales, Universitat Politècnica de València, Camino de Vera s/n, 46022 Valencia, Spain

Email address: mjrupere@upvnet.upv.es (M.J. Rupérez)
} 
Keywords: Hip fracture, clinical, osteoporosis, finite element, machine learning

\section{Introduction}

Osteoporotic hip fracture is a major health problem and tends to become more prevalent as the population ages $[1,2]$. By 2050, the worldwide annual incidence could reach 6.26 million [3] and the associated burden on the health system is estimated to be $\$ 131.5$ billion [4]. For each patient, collateral effects may include increased risk of mortality, disability, chronic pain and reduced quality of life [5]. For this, a comprehensive assessment of relevant predictors is necessary to accurately identify patients at higher risk and reduce the incidence through patient-specific interventions $[6,7]$.

Osteoporosis is a skeletal disease primarily characterized by reduced bone mass [8]. Bone mineral density, measured by Dual-Energy X-Ray Absorptiometry, is the gold standard in current clinical practice for osteoporosis diagnosis and hip fracture risk assessment [9]. However, its discrimination ability between fractured and control cases is limited. BMD distributions for age-matched samples of both groups overlap for large amount, reducing the classification accuracy to about $65 \%$ [10]. On the other hand, quantitative computed tomography (QCT) allows to obtain three-dimensional geometry of the bone and provides with volumetric distribution of BMD (vBMD), which is considered to be more sensitive for osteoporosis [11, 12]. Nevertheless, QCT is not integrated in clinical routine due to its higher cost, processing time and radiation exposure [13].

Alternative methods have been proposed to obtain better predictors. Finite Element (FE) models based on DXA include both BMD distribution and twodimensional proximal femur architecture in order to quantify the mechanical strength of the bone. These models have demonstrated to provide patientspecific estimates of strength and moderately increase the classification accuracy $[14,15,16]$. These models are considered to be not as accurate as QCT-based models $[17,18]$, but, from the above reasons DXA models are highly attractive for clinicians as the current clinical workflow is not interrupted . Furthermore, 
fracture risk and Hip Structural Analyses (HSA) derived from both QCT and DXA based models have been found to be significantly correlated [19], and DXA models highly reduced the computational cost.

Statistical models have been developed to calculate the risk of fracture relying on clinical factors. The fracture risk assessment tool (FRAX) and the Garvan tool are the most popular ones [20,21]. Patient data are compared to a large database that includes many features, e.g. age, gender, previous fractures, etc. The classification accuracy is similar for both, about $70 \%$ [22] and there is not a significant improvement compared to BMD. Likewise, machine learning (ML) techniques have risen as a suitable way of analysing complex data and extracting unexpected risk factors for the field of preventive medicine.

Regarding osteoporotic hip fracture, some studies have collected a large dataset and have combined several clinical attributes and DXA-derived measurements in different classification models to outperform the predictive power of BMD $[23,24]$. These studies did not include mechanical attributes into their models and were limited by their interpretability. Combination of clinical and mechanical attributes has been most commonly addressed in the literature by FE approaches. However, the majority of these studies did not explore ML classifiers beyond Logistic Regression. A recent study aimed to use high-resolution MRI derived data to compare $15 \mathrm{ML}$ classifiers performance at predicting any kind of osteoporotic fracture [25]. The data comprised bone tissue elasticity and topology of the proximal femur, at specific volumes of interest, computed with micro-finite elements. Although this study gave insight into the relevance of microstructural parameters, the dataset was small and was not specially focused on hip fracture.

On the other hand, patient-specific QCT-based FE analyses under multiple loading conditions were used as input for a Support Vector Machine (SVM) classifier to address uncertainty in the fall configuration [26]. Other authors investigated the combination of clinical and FE-derived mechanical attributes by means of SVM augmented space [27]. However, their parametrized FE model did not include patient-specific data describing the geometry nor the BMD dis- 
tribution. Even though, the results showed that including mechanical attributes into a SVM model significantly enhanced hip fracture prediction rather than using clinical attributes alone.

In this work, we propose a novel methodology in which a semi-automatic and patient-specific 2D Finite Element (FE) model based on Dual-Energy X-Ray Absorptiometry (DXA) is used to generate mechanical attributes that will serve as input data for a ML classifier, together with basic clinical information. We hypothesized that combining the predictive power of each attribute would improve the accuracy of hip fracture classification compared to the gold-standard Bone Mineral Density (BMD) and other clinical data alone. Additionally, the study aimed to identify the best-learned ML classifier within a group of selected algorithms suitable for this problem.

\section{Material and methods}

\subsection{Study population}

A total number of 137 patients were included into the study with a mean age of $81.4 \pm 6.95$ years. Patients were separated into a fracture group $(\mathrm{n}=89)$, with fall-related incident hip fracture, and a control group $(n=48)$. Within the fracture group, 45 accounted for a trochanteric fracture and 44 for a neck fracture. Inclusion criteria comprised: postmenopausal women, older than 50 years, clinical risk factors related to osteoporosis and no artefacts within the image.

The scans were performed at CETIR Medical Group, after informed consent. The time between fracture and DXA acquisition was less than two weeks. DXA scans were taken on the opposite femur to the fracture using GE Healthcare Prodigy Advance bone densitometer (GE Healthcare, Madison, WI, USA). The image pixel size in these scans was $0.6 \mathrm{~mm} \times 1.05 \mathrm{~mm}$.

\subsection{Automated patient-specific FE model}

For the purpose of determining mechanical attributes, a 2D patient-specific FE model, was created using a set of MATLAB (The Mathworks Inc., Natick, 


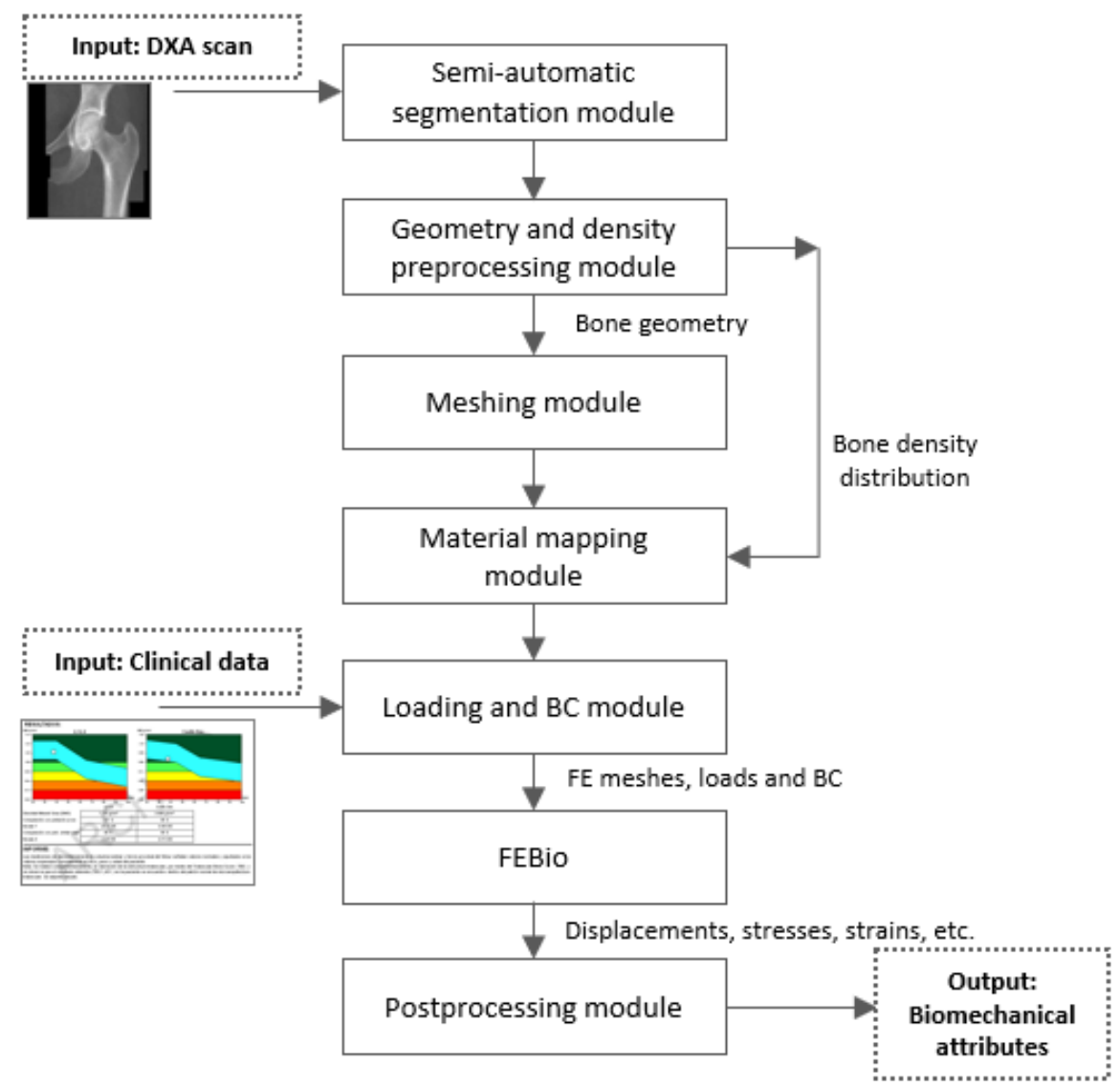

Figure 1: Workflow of the implemented patient-specific FE model

MA, USA) in-house code. A workflow diagram is provided in Figure 1. The model was built under the assumptions of plane strain and linear elasticity behaviour. Previous studies developed DXA-based models following a plane stress approach based on the femur dimensions [28, 16]. However, the authors consider that plane strain is more suitable in the anterior and posterior plane, as the $\mathrm{Z}$ dimension (perpendicular to the plane) is not negligible. The open-source FE package FEBio [29] was used to obtain the numerical solution. The only input required for the construction of the FE model was the segmented femur taken from the DXA scan (Figure 2), along with the basic clinical information of the patient (height, weight and gender). 


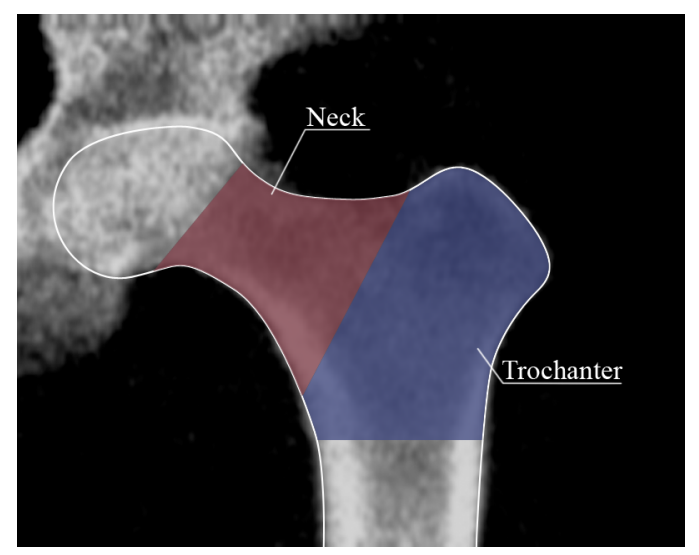

Figure 2: Proximal femur, neck region and trochanter region segmentation

For each DXA scan, the proximal femur was segmented manually. Regions of interests (ROIs) defining the trochanteric and neck region were defined semiautomatically (Figure 2). After that, the implemented workflow was completely automated. Pads covering the femoral head and the greater trochanter were placed to avoid local damage due to the applied boundary conditions [30, 31]. Finally, the femur shaft was rotated 10 degrees to the physiological configuration.

In the meshing module, the open-source mesh generator TetGen [32] was integrated. The generated mesh was separated into three regions: the femur, the trochanteric pad and the femoral head pad. Following a convergence analysis, the mesh size was defined. For this, each mesh had approximately 60,000 elements, depending on the subject.

Bone material properties were derived from the BMD map within the image. The relationship between grey values and areal Bone Mineral Density (aBMD) was obtained using the values measured by the DXA scan software and reverse engineering techniques. The mean BMD value at the proximal femur (Total BMD) is available in the subject's clinical records and its measurement process is known from the literature [9]. A semi-automatic measurement method to reproduce this process was developed. The mean grey value at the specific ROI was obtained and correlated with the clinical record BMD values at the same 
site. A linear relationship was obtained $\left(\mathrm{n}=40, R^{2}=0.988, p\right.$-value $\left.<0.001\right)$. Afterwards, aBMD of each pixel was mapped into the finite element mesh using this relationship, as seen in Figure 3a. The femur was assumed to have a constant subject-specific thickness ( $\mathrm{t}$ ) for the transverse direction [33, 28]. For this, the area and moment of inertia were considered to be closest to that of a circular section, at a cross-section in the middle femoral neck (with a width NW) . This way, volumetric BMD (vBMD) was calculated as the division between aBMD and $t$. For each element in the mesh, vBMD was converted to ash density $\left(\rho_{\text {ash }}\right)$ and to apparent density $\left(\rho_{\text {app }}\right)$, according to previous studies [34]. Thus, the apparent density could be calculated through equation (1):

$$
\rho_{a p p}=\frac{a B M D}{1.14 \cdot 0.598 \cdot t}
$$

Then, bone material properties were calculated using the empirical equations obtained by Morgan and colleagues [35, 36, 37]:

$$
\begin{gathered}
E=\left\{\begin{array}{ll}
15010 \rho_{\text {app }}^{2.18} & \text { if } \rho_{\text {app }} \leq 0.280 \mathrm{~g} / \mathrm{cm}^{3} \\
6850 \rho_{\text {app }}^{1.49} & \text { if } \rho_{\text {app }}>0.280 \mathrm{~g} / \mathrm{cm}^{3}
\end{array}\right\} \\
S_{y c}=\left\{\begin{array}{ll}
85.5 \rho_{\text {app }}^{2.26} & \text { if } \rho_{a p p} \leq 0.355 \mathrm{~g} / \mathrm{cm}^{3} \\
38.5 \rho_{\text {app }}^{1.49} & \text { if } \rho_{a p p}>0.355 \mathrm{~g} / \mathrm{cm}^{3}
\end{array}\right\} \\
S_{y t}=\left\{\begin{array}{ll}
50.1 \rho_{\text {app }}^{2.04} & \text { if } \rho_{a p p} \leq 0.355 \mathrm{~g} / \mathrm{cm}^{3} \\
22.6 \rho_{\text {app }}^{1.26} & \text { if } \rho_{a p p}>0.355 \mathrm{~g} / \mathrm{cm}^{3}
\end{array}\right\} \\
E_{y c}=\left\{\begin{array}{ll}
0.7 & \text { if } \rho_{a p p} \leq 0.27 \mathrm{~g} / \mathrm{cm}^{3} \\
0.85 & \text { if } \rho_{a p p}>0.27 \mathrm{~g} / \mathrm{cm}^{3}
\end{array}\right\}
\end{gathered}
$$

Where $\mathrm{E}$ is the Modulus of Elasticity, $\mathrm{S}_{\mathrm{yc}}$ is the Compressive Yield Stress and $\mathrm{S}_{\mathrm{yc}}$ is the Tensile Yield Stress, all of them in MPa. The Compressive Yield Strain 
$\mathrm{E}_{\mathrm{yc}}$ was expressed in hundred percent (\%). These variables were adjusted by a factor of 1.28 to account for side-artefact errors that occur in the biomechanical testing of cadaveric bone used to establish those equations [38]. As a result, bone tissues were modelled as linear isotropic elastic materials [39] and Poisson's ratio was set to 0.3 [40]. PMMA (Polymethylmethacrylate) material properties were considered for the pads (1.5 GPa for the Elastic Modulus and 0.37 for the Poisson's ratio), similar to previous studies involving mechanical tests [41] and FE models [42]. Finally, a heterogeneous material distribution was obtained, as shown in Figure 3b for the Young's Modulus.

The boundary conditions (BC) applied to the femur simulated a sideways-fall onto the greater trochanter (Figure 3c). Several studies investigated the influence of different boundary conditions in the stress-strain pattern of the bone $[43,44]$. On the basis of these studies, the nodes at the distal end of the femoral shaft were fixed and medial displacement of the nodes at the femoral head pad was prevented (Figure 3c). The load was applied to the greater trochanter through its pad, representing the fall-related impact force. The load was calculated by means of the peak impact force obtained with a mass-spring impact model [45].

For this model, the load calculation scheme had as input variables the weight, height and gender of the patient. The two-linked 45 degrees Jack-knife model was implemented for the calculation of the impact velocity and the three-linked 3C model was implemented for the effective mass at impact. Segments properties distribution and angles at impact configuration were taken from the same study. A value for the stiffness constant $(\mathrm{K})$ of $71,060 \mathrm{~N} / \mathrm{m}$ was considered according to experiments in human volunteers [46]. Once the peak impact force (FPK) was derived, the attenuated impact force (FP) was calculated considering the attenuation force $(F A T=71 \cdot S T H)$, on the basis of previous published studies regarding the effect of soft tissue thickness (STH) [47] and correlations between body mass index (BMI) and STH [48].

$$
F P=F P K-71 \cdot S T H
$$




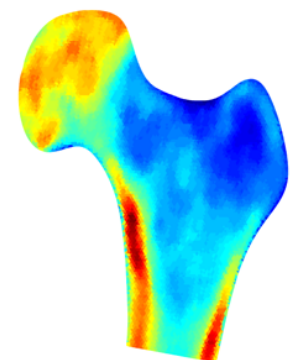

(a) BMD mapping obtained from the DXA scan

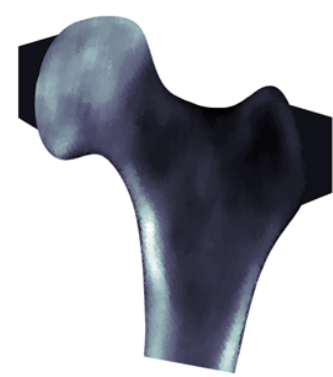

(b) Young's Modulus map

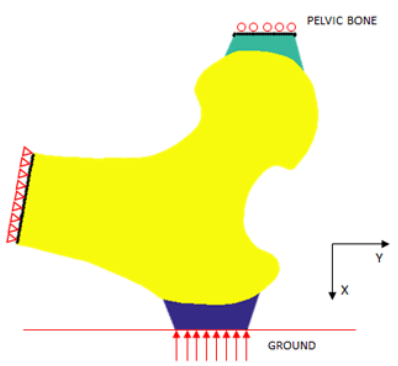

(c) Boundary conditions simulating a sideways fall impact at the greater trochanter

Figure 3: Material properties, loading and boundary conditions automatically generated

The applied load pressure (HP) was computed dividing the attenuated impact force by the length of the greater trochanter pad (b) and the subject-specific 


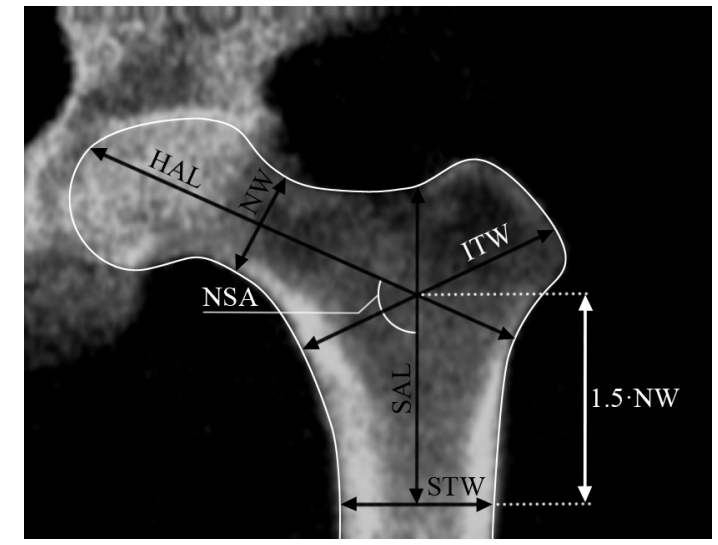

Figure 4: Graphical description of geometrical attributes

thickness ( $\mathrm{t})$ :

$$
H P=\frac{F P}{b \cdot t}
$$

\subsection{Input parameters to the predictive model}

For each sample, we have collected five groups of attributes. The first group was formed by attributes that are usually collected in clinical practice and the rest were formed by attributes involved in the Finite Element Analysis (Geometrical, Fall-related, Bone density and FEA-derived). Table 1 shows each attribute together with their mean and standard deviation.

Geometrical attributes were obtained through a morphometric analysis performed on the proximal femur geometry [16]. Specifically, neck width (NW), hip axis length (HAL), neck-shaft axis angle (NSA), shaft axis length (SAL), intertrochanteric width (ITW) and subtrochanteric width (STW). Additionally, the proximal femur area (FA) was computed. The general scheme to obtain these attributes is shown in Figure 4.

Regarding the fall-related attributes, impact force (FP); peak impact force (FPK); attenuation force (FAT); soft tissue thickness (STH); body mass index 
(BMI) and the hip pressure (HP) were computed for each patient.

As bone tissue structural properties are inherently an important factor related to bone fracture, we also decided to obtain attributes that represented these properties. Cortical bone was defined as having an apparent density greater than $1.0 \mathrm{~g} / \mathrm{cm}^{3}$ [42]. We estimated the percentage of trabecular bone (TB) and cortical bone (CT) within the femur bone using this threshold and computed the average Young's Modulus within each tissue (TBV and CTV).

From the FE linear analysis performed with FEBio, several mechanical attributes were extracted for each patient. The Load-to-Strength-Ratio (LSR) and the Femoral Strength (FS) were computed to define the failure of the whole bone [17]. For this, a contiguous area of $9 \mathrm{~mm}^{2}$, where the elements with highest ratios between the Principal Compressive Strain and the Eyc was identified. LSR was defined as the minimum ratio in this area. Yet, the femur is a heterogeneous biological structure that can fracture in multiple sites, being the most common the neck and the trochanteric regions. For this, it was decided to also obtain mechanical attributes at each region (index $\mathrm{N}$ will be used for the variables at neck region, and index $\mathrm{T}$ for the variables at the trochanter region). The volume weighted average value of maximum and minimum principal stress $\left(\mathrm{S}_{1}\right.$ and $\left.\mathrm{S}_{3}\right)$, maximum and minimum principal strain $\left(\mathrm{E}_{1}\right.$ and $\left.\mathrm{E}_{3}\right)$, major principal stress (MPStress), major principal strain (MPStrain), strain energy density (SED) and the fracture risk index (FRI) were computed. MPStress and MPStrain were defined as the maximum eigenvalue in the stress and strain tensor, respectively. FRI was computed as the weighted average ratio between the Von Mises stress and the yield stress in the region. The yield stress was computed from equation (3) and (4), depending on the dominant principal stress in the element.

Table 1: Descriptive statistics for baseline attributes used in this study

\begin{tabular}{lccc}
\hline Category & Attributes & $\begin{array}{c}\text { Fractured (mean } \pm S D) \\
(\mathrm{N}=89)\end{array}$ & $\begin{array}{c}\text { Control }(\text { mean } \pm S D) \\
(\mathrm{N}=48)\end{array}$ \\
\hline Clinical & Age (years) & $81.39 \pm 6.98$ & $82.56 \pm 3.88$
\end{tabular}


Table 1 continued...

\begin{tabular}{|c|c|c|c|}
\hline \multirow[t]{6}{*}{ Category } & Attributes & Fractured $($ mean $\pm S D)$ & Control (mean $\pm S D)$ \\
\hline & Height $(\mathrm{cm})$ & $152.67 \pm 7.09$ & $151.75 \pm 5.09$ \\
\hline & Weight (kg) & $63.61 \pm 14.03$ & $65.21 \pm 10.01$ \\
\hline & BMI $\left(\mathrm{kg} / m^{2}\right)$ & $27.28 \pm 5.70$ & $28.31 \pm 4.02$ \\
\hline & BMD_total & $0.70 \pm 0.13$ & $0.80 \pm 0.10$ \\
\hline & $\left(\mathrm{g} / \mathrm{cm}^{2}\right)$ & & \\
\hline \multirow[t]{7}{*}{ Geometrical } & $\mathrm{NW}(\mathrm{mm})$ & $29.29 \pm 2.02$ & $29.68 \pm 2.32$ \\
\hline & HAL (mm) & $89.74 \pm 6.31$ & $88.98 \pm 5.48$ \\
\hline & $\operatorname{NSA}\left({ }^{\circ}\right)$ & $126.48 \pm 6.11$ & $124.21 \pm 6.24$ \\
\hline & FA $\left(m m^{2}\right)$ & $4580.09 \pm 490.35$ & $4574.10 \pm 372.61$ \\
\hline & $\mathrm{SAL}(\mathrm{mm})$ & $83.16 \pm 5.34$ & $85.79 \pm 5.19$ \\
\hline & ITW (mm) & $50.29 \pm 3.12$ & $50.15 \pm 3.32$ \\
\hline & STW (mm) & $27.65 \pm 2.17$ & $26.44 \pm 1.52$ \\
\hline \multirow[t]{5}{*}{ Fall-related } & $\mathrm{STH}(\mathrm{mm})$ & $30.43 \pm 13.43$ & $32.85 \pm 9.42$ \\
\hline & FPK $(\mathrm{N})$ & $5206.08 \pm 641.10$ & $5284.01 \pm 455.86$ \\
\hline & FAT $(\mathrm{N})$ & $2160.27 \pm 947.06$ & $2332.19 \pm 668.80$ \\
\hline & $\mathrm{FP}(\mathrm{N})$ & $3045.81 \pm 518.10$ & $2951.82 \pm 371.04$ \\
\hline & $\mathrm{HP}(\mathrm{MPa})$ & $6.74 \pm 1.14$ & $6.46 \pm 0.87$ \\
\hline \multirow[t]{4}{*}{ Bone density } & TB (\%) & $86.94 \pm 9.04$ & $82.10 \pm 8.92$ \\
\hline & TBV (GPa) & $3.59 \pm 0.53$ & $3.85 \pm 0.39$ \\
\hline & $\mathrm{CT}(\%)$ & $13.06 \pm 9.04$ & $17.90 \pm 8.92$ \\
\hline & CTV (GPa) & $10.89 \pm 1.96$ & $11.57 \pm 1.00$ \\
\hline \multirow[t]{14}{*}{ FEA-derived } & LSR & $0.98 \pm 0.65$ & $0.63 \pm 0.28$ \\
\hline & $\mathrm{FS}(\mathrm{N})$ & $4421.34 \pm 2553.70$ & $5285.70 \pm 1718.72$ \\
\hline & $\mathrm{S} 1 \_\mathrm{N}(\mathrm{MPa})$ & $1.49 \pm 0.40$ & $1.46 \pm 0.36$ \\
\hline & S3_N (MPa) & $-3.88 \pm 0.77$ & $-3.81 \pm 0.68$ \\
\hline & E1_N ( $\mu$ strain) & $1042.10 \pm 430.64$ & $816.49 \pm 180.71$ \\
\hline & $\mathrm{E} 3 \_\mathrm{N}(\mu$ strain $)$ & $-2250.30 \pm 1016.03$ & $-1689.79 \pm 400.57$ \\
\hline & MPStress_N (MPa) & $5.15 \pm 1.07$ & $5.12 \pm 0.94$ \\
\hline & MPStrain_N & $2353.53 \pm 1034.01$ & $1784.85 \pm 414.42$ \\
\hline & ( sstrain) & & \\
\hline & SED_N $\left(J / m^{3}\right)$ & $7473.06 \pm 4113.33$ & $5669.81 \pm 1903.91$ \\
\hline & S1_T (MPa) & $0.40 \pm 0.13$ & $0.38 \pm 0.11$ \\
\hline & $\mathrm{S} 3 \_\mathrm{T}(\mathrm{Pa})$ & $-2.94 \pm 0.55$ & $-2.84 \pm 0.49$ \\
\hline & E1_T ( $\mu$ strain $)$ & $689.95 \pm 363.99$ & $499.23 \pm 124.25$ \\
\hline & E3_T ( $\mu$ strain) & $-1457.57 \pm 875.67$ & $-1105.74 \pm 273.43$ \\
\hline
\end{tabular}


Table 1 continued...

\begin{tabular}{llcc}
\hline Category & \multicolumn{1}{c}{ Attributes } & Fractured $($ mean $\pm S D)$ & Control $($ mean $\pm S D)$ \\
\hline MPStress_T $(\mathrm{MPa})$ & $3.24 \pm 0.59$ & $3.12 \pm 0.52$ \\
MPStrain_T & $1571.41 \pm 883.43$ & $1122.79 \pm 276.95$ \\
$(\mu$ strain $)$ & & \\
SED_T $\left(\mathrm{J} / \mathrm{m}^{3}\right)$ & $3536.94 \pm 2194.64$ & $2540.23 \pm 896.58$ \\
FRI_N & $0.32 \pm 0.14$ & $0.24 \pm 0.06$ \\
FRI_T & $0.21 \pm 0.12$ & $0.15 \pm 0.04$ \\
\hline
\end{tabular}

All these predictor attributes were used to predict the dependent variable Group that indicates if the sample belongs to the fractured group (Fracture) or the control group (Control). In total, we had 39 attributes and 137 samples. Therefore, we had an excess of attributes for a so small cohort. We decided to keep only the ones with Pearson correlation lower than 0.8. Figure 5 shows a heat map of the correlation matrix of the attributes described in Table 1. As Figure 5 shows, there were groups of attributes (like FRI_T, SED_T, MPStraint_T and E3_T) that were highly correlated. In these cases, keeping one representative of each group is enough to learn an appropriate model. Table 2 shows the 19 predictor attributes left after removing the highly correlated ones.

Besides, the mentioned predictor attributes, in some cases, presented differences of several orders of magnitude. To avoid bias due to these differences, we standardized all of them (Z-score normalization). 
Table 2: Non-correlated predictor attributes

\begin{tabular}{cccccc}
\hline & Clinical & Geometrical & Fall-related & Bone density & FEA-derived \\
\hline \multirow{4}{*}{ Age } & NW & HP & TB & LSR \\
& Height & HAL & & CTV & FS \\
& Weight & NSA & & & S1_N \\
& BMD_total & SAL & & & E1_N \\
& & ITW & & S1_T \\
& & STW & & SED_T \\
\hline
\end{tabular}

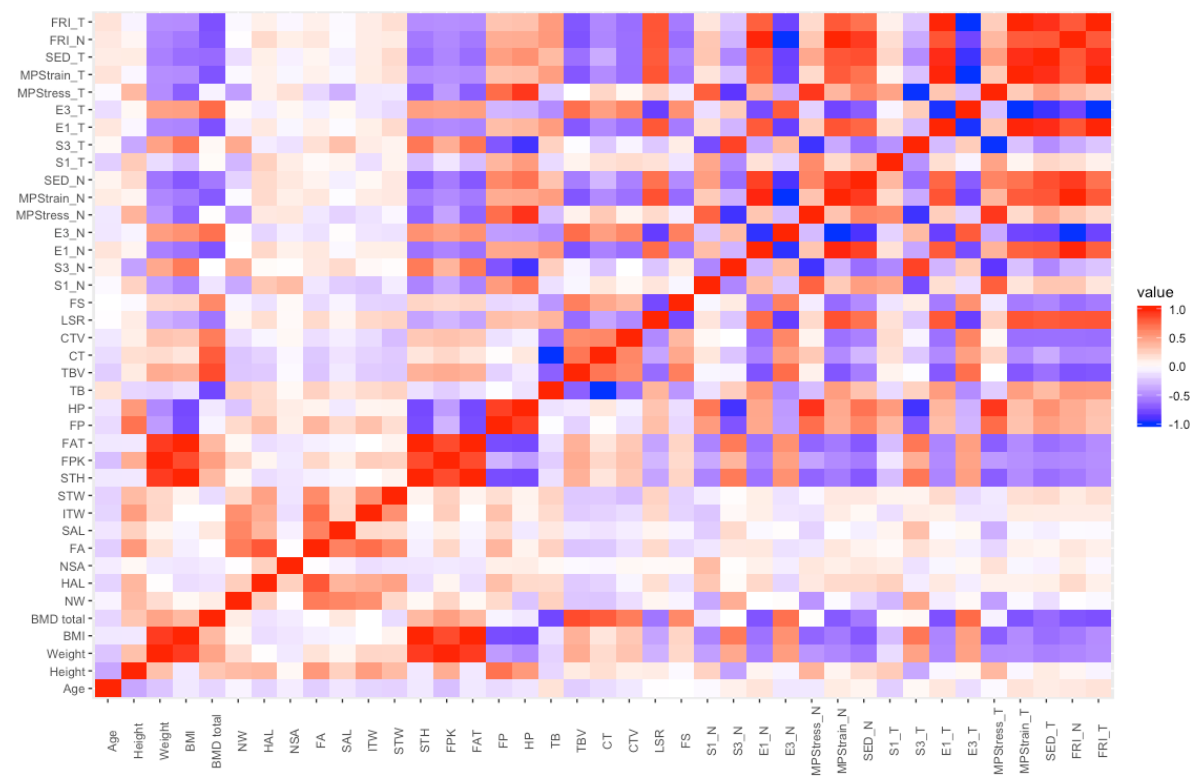

Figure 5: Heat map of the correlation matrix between attributes

\subsection{Machine Learning Procedure}

To look for the best classification algorithm, we compared the results of a set of algorithms that fitted the characteristics of the problem like Support Vector Machine (SVM) with radial basis function (RBF), Logistic Regression, Shallow Neural Networks with only a hidden layer of 10 nodes and Random Forest. For model building and evaluation, we decided to divide the samples in two sets: 101 for training and 36 for test. These sets were built by taking random 
samples without replacement. Besides, 10 fold cross-validation was applied for the training set in order to obtain the model.

Once the model was built, we checked its prediction capability with the test set formed by samples unseen during the model construction. All this process was repeated four times to avoid overfitting because of the reduce volume of samples. Therefore, we built 16 models that were checked with the corresponding test sets. Besides, to check the improvement introduced by the learned models, we compared with a dummy model that assigned all samples to the majority class.

Finally, to analyse if the use of Finite Element attributes improved the results of the learned models, we performed the same experiments but, in this case, using only the clinical attributes. This implied the building of 16 new models that, again, were checked with the corresponding test sets.

\section{Results}

Table 3 shows the results of the described experiments conducted using 19 attributes. This table shows the percentage of accuracy in training and test for each model in each experiment. On average, all the learned models are over the performance of the dummy model. However, only the SVM is over the performance of the dummy model in all the experiments. Besides, although SVM is slightly under the average performance of Logistic Regression and Neural Network during the training experiments, the mean of SVM outperforms the

others in the test experiments. These results are also shown in Figure 6. This way, SVM was confirmed to generate the best-learned model for our problem (78.35\% accuracy at test).

For the purpose of evaluating the contribution of the patient-specific biomechanical attributes, we repeated the same experiments including only clinical attributes in the model. Table 4 and Figure 7 show the results. These results are worse than the ones obtained using all the attributes. In this case, on average, SVM model was slightly under the Logistic Regression performance, but it 
Table 3: Analysis of the learned models using 19 attributes. Values represent the percentage of accuracy at each experiment for the training and test datasets

\begin{tabular}{l|cc|cc|cc|cc|cc}
\hline & \multicolumn{2}{|c|}{ Logistic Reg. } & \multicolumn{2}{|c|}{ SVM } & \multicolumn{2}{|c|}{ Neural Network } & \multicolumn{2}{c}{ Random Forest } & \multicolumn{2}{c}{ Majority class } \\
\hline & Training & Test & Training & Test & Training & Test & Training & Test & Training & Test \\
\hline $\mathbf{1}$ & 83.17 & 66.67 & 72.28 & 86.11 & 77.22 & 83.33 & 63.37 & 83.33 & 65.35 & 63.89 \\
$\mathbf{2}$ & 81.18 & 66.67 & 80.20 & 75.00 & 81.18 & 58.33 & 70.30 & 66.67 & 61.39 & 75.00 \\
$\mathbf{3}$ & 66.34 & 88.89 & 74.26 & 77.78 & 74.26 & 72.22 & 76.24 & 69.44 & 62.38 & 72.22 \\
$\mathbf{4}$ & 77.23 & 72.22 & 76.24 & 75.00 & 73.27 & 69.44 & 68.32 & 75.00 & 65.35 & 64.00 \\
\hline Average & 76.69 & 73.09 & 75.69 & 78.35 & 76.42 & 70.26 & 69.40 & 73.34 & 63.59 & 68.60
\end{tabular}

Table 4: Analysis of the learned models using clinical attributes. Values represent the percentage of accuracy at each experiment for the training and test datasets

\begin{tabular}{l|cc|cc|cc|cc|cc}
\hline & \multicolumn{2}{|c|}{ Logistic Reg. } & \multicolumn{2}{|c|}{ SVM } & \multicolumn{2}{|c|}{ Neural Network } & \multicolumn{2}{c|}{ Random Forest } & \multicolumn{2}{c}{ Majority class } \\
\hline & Training & Test & Training & Test & Training & Test & Training & Test & Training & Test \\
\hline $\mathbf{1}$ & 70.30 & 72.22 & 71.30 & 69.44 & 66.34 & 72.22 & 68.32 & 69.44 & 65.35 & 63.89 \\
$\mathbf{2}$ & 74.26 & 69.44 & 68.32 & 75.00 & 68.32 & 64.00 & 67.33 & 69.44 & 61.39 & 75.00 \\
$\mathbf{3}$ & 65.35 & 86.11 & 64.36 & 75,00 & 68.32 & 72.22 & 66.34 & 77.78 & 62.38 & 72.22 \\
$\mathbf{4}$ & 69.31 & 63.89 & 72.28 & 69.44 & 68.32 & 66.67 & 71.29 & 63.89 & 65.35 & 64.00 \\
\hline Average & 69.73 & 72.47 & 68.99 & 72.17 & 67.82 & 68.68 & 68.29 & 69.96 & 63.59 & 68.60
\end{tabular}

was the only one with all results better than the dummy model. Hence, SVM was confirmed again to generate the best-learned model (72.17\% accuracy).

Additionally, we compared our classification model to a Logistic Regression model built with BMD alone, following the same training and test procedure as before. We measured the accuracy metrics of this model, this giving rise to $66.81 \%$ accuracy at training and $64.88 \%$ accuracy at test (Figure 8 ), which are similar values to those found in the literature [10]. This means that our best-learned model (SVM) has improved the gold-standard performance by approximately $14 \mathrm{pp}$. The results showed that hip fracture prediction can be significantly enhanced beyond basic clinical attributes (including the gold-standard BMD) by means of patient-specific biomechanical attributes and SVM. 


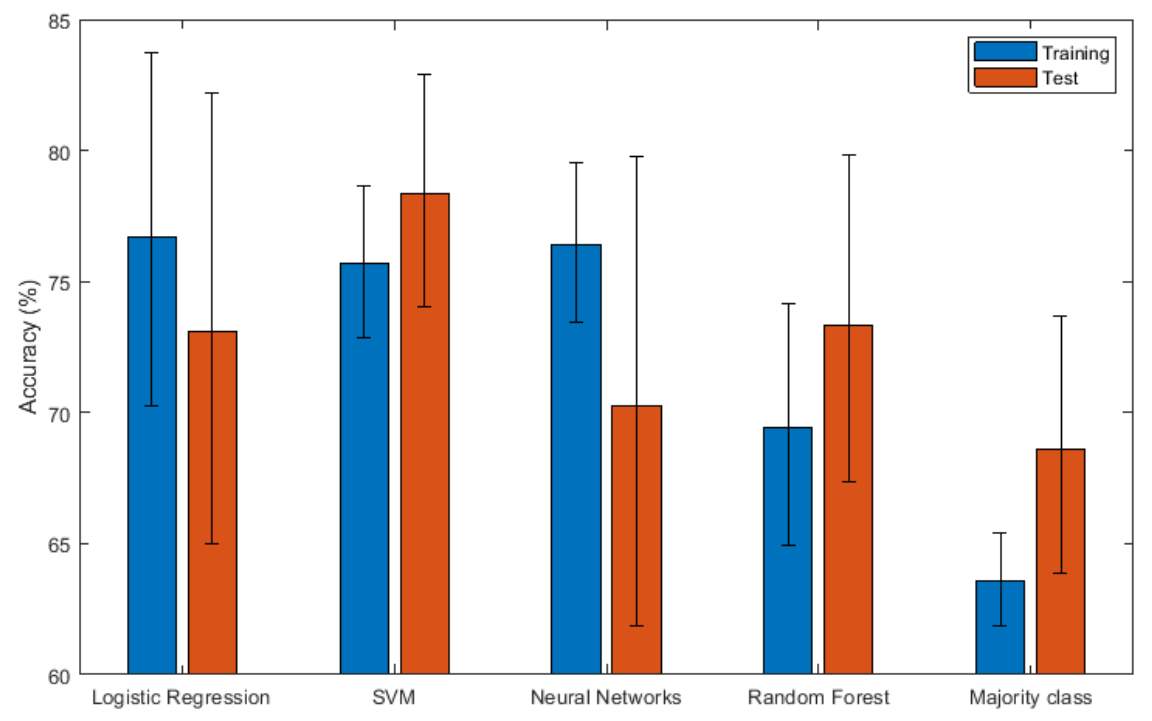

Figure 6: Accuracy for each trained and tested classification model when including 19 attributes $($ Mean $\pm 1 S D)$ 


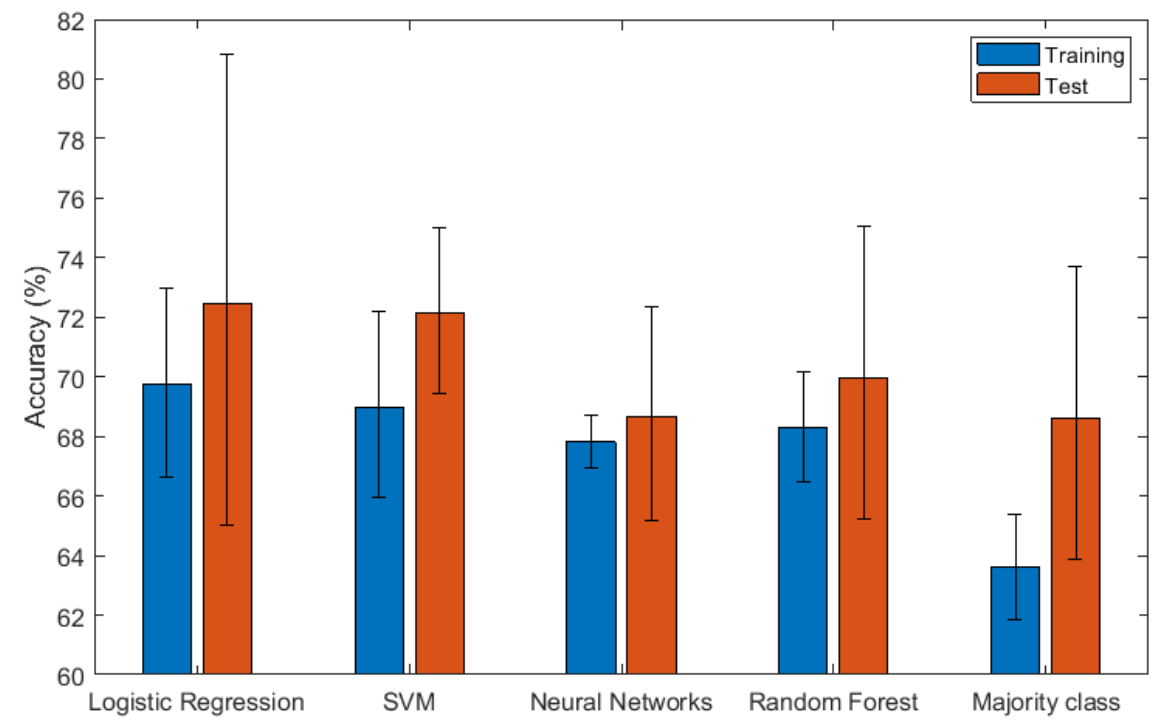

Figure 7: Accuracy for each trained and tested classification model using only clinical attributes $($ Mean $\pm 1 S D)$

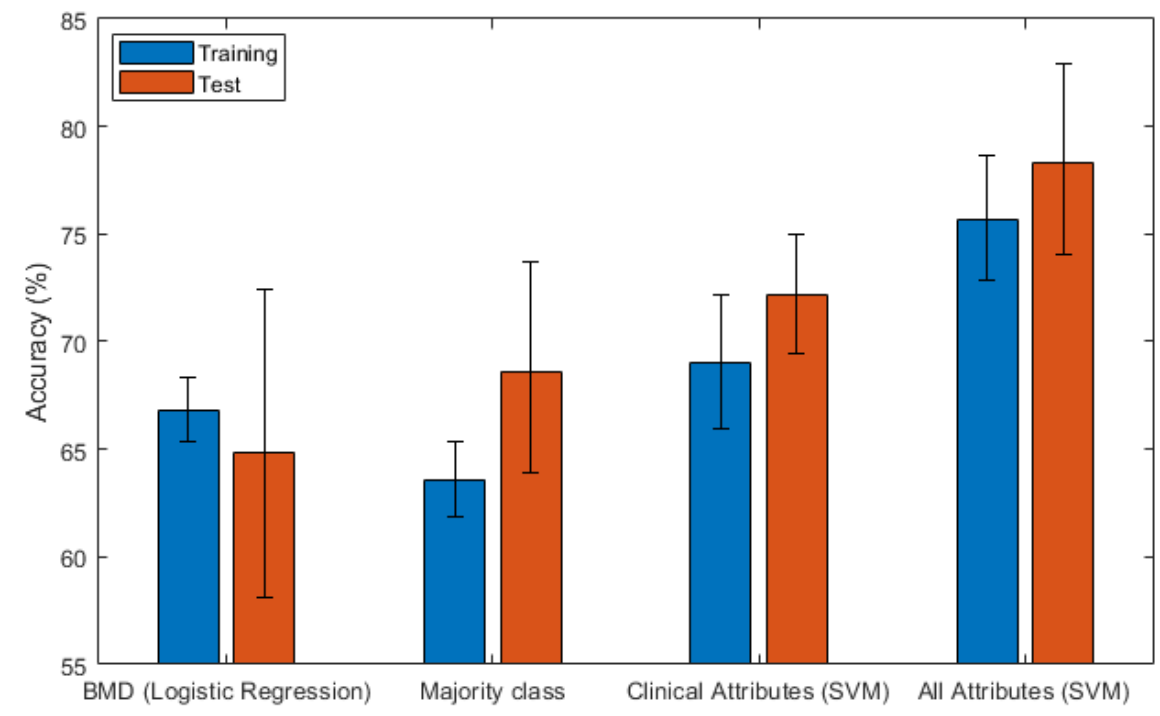

Figure 8: Benchmark for BMD vs. learned algorithms (Mean $\pm 1 S D)$ 


\section{Discussion}

To the best of our knowledge, we have presented the first approach to combine clinical and 2D patient-specific biomechanical data through different Machine Learning classification algorithms to predict hip fracture. A previous work attempted to combine clinical and 3D FEA-derived data with SVM [27]. In this study, the parametrized FE model was based in a stochastic approach and did not include patient-specific information from images describing the BMD distribution, the geometry or the impact force. However, it is discussed about the possibility of using multiple failure criteria in conjunction, which we have applied to our model. Specifically, we have considered 9 different failure criteria, due to the existing lack of agreement on a failure criterion for bone tissue [49]. We have considered two whole bone failure attributes (LSR and FS), whereas we have considered 8 site-specific failure attributes for the neck site (e.g. S1_N, S3_N,...) and the trochanter site (e.g. S1_T, S3_T,...). Site-specific attributes were computed due to the difference in the etiology for fractures at both sites [50]. After preprocessing the data, we found that 4 site-specific attributes and the LSR were enough to describe the information obtained from the FE solution. Moreover, we found that removing any of these attributes would decrease the accuracy of our SVM classifier. This suggests that the multi-criteria approach may improve hip fracture prediction and should be further explored.

Most of previous studies involving patient-specific FE modelling only focused to predict hip fracture through univariate or multivariate linear classification models. Although this kind of models are easy to implement, they may lead to an underestimation of the predictive capabilities of the studied attributes, as the decision boundary between classes (fractured and controls) might be non-linear. For the linear approach, it has been reported a marginal increase in accuracy metrics, compared to BMD, of 4-5pp when using the FE attributes alone [33, 51]. In this context, others found an increase of $17 \mathrm{pp}$ when integrating geometry, height and femoral neck BMD together with a FE attribute [52], but the sample size was relatively small and only included neck fractures. Nevertheless, this 
reinforced the idea that $\mathrm{FE}$ attributes should be combined with other kind of attributes to enhance hip fracture prediction. This has been successfully implemented in our current study for a wider sample population, using more sophisticated classification methods. In fact, we found that accuracy metrics at test for linear models as Logistic Regression were considerably lower than for our best-learned model (SVM), which is non-linear, when including FEAderived attributes. This also suggests that non-linear functions may be required in order to find an optimal decision boundary when including FEA attributes into the learning procedure.

We have used as input patient-specific data describing the two-dimensional geometry, the BMD distribution and loading conditions and built a linear elastic finite element model. In this sense, we acknowledge the inherent limitations of a $2 \mathrm{D}$ model at describing the mechanical response of bone tissue. Bone is a three-dimensional heterogeneous and anisotropic structure subjected to complex loading states. A better representation of bone shape and density distribution could be obtained through 3D reconstruction from 2D images [53, 54]. This would most likely enhance the FEA-derived attributes and increase the classification accuracy of the ML model. However, solving a 3D FE model can be computationally demanding, whereas our FE model takes 2 seconds to be solved. Besides, 3D models are expensive because it requires 3D CTs or MRIs that are out of the actual clinical workflow. Therefore, the approach shown in this study is more feasible to obtain fair estimates of patient-specific bone mechanical attributes and drive a ML classifier during routine clinical practice.

The described methodology was applied to an age-matched population of postmenopausal women, where the fractured group $(n=89)$ accounted for a time to fracture of less than 2 weeks. This is one of the strengths of our study. It means that the BMD maps of our fractured patients were close to the fracture instant, thus, less influenced by bone remodelling than other studies found in the literature. Another strength is that the population included in the study accounted for both neck and trochanteric fracture in a similar proportion, which are the main types of hip fracture. This means that our methodology can predict 
the fracture event, no matter the fracture location. In addition, a comprehensive validation procedure was used in order to avoid bias due to the modest sample size. This helped to find the Machine Learning algorithm with the best biasvariance trade-off for our problem.

The main limitation of the present study was the sample size and the image acquisition. Although the sample size was larger than other studies, it was still modest and this what limited the learning process. On the other hand, pixel surface was approximately 8 times greater than in other commercial densitometers (e.g. GE Healthcare iDXA Advance), thus, having significantly less resolution. As the outcome of the FE analyses highly depend on the material properties assigned to the mesh, the discriminative power of FEA-derived attributes rely on how detailed BMD maps are. The authors have performed a preliminary check on the identification of the fracture type using the LSR attribute, this driven similar conclusions to that of previous studies $[28,16]$. The correct identification of fracture type is a challenge for $2 \mathrm{D}$ finite analyses due to the inherent 2D limitation and the overlapping of cortical and trabecular bone on the image plane. Therefore, stress and strain distributions may be altered, and the failure starting location might not be fully reliable. This challenge has remained unfulfilled in the literature due to the mentioned limitations. In our study, due to the limited amount of data that is obtained after splitting the dataset considering the fracture type, we could not differentiate in our learned models between neck and trochanteric fractures. Finally, although our study focused on prediction of hip fracture in postmenopausal women, hip fracture also occur in the male population. Moreover, differences between males and females fracture attributes, both clinic and biomechanical, has been shown in the literature $[55,56]$. This should be addressed in order to build effective predictive models for both genders. 


\section{Conclusions}

This study showed that hip fracture prediction can be modeled and improved by a multi-technique approach, considering clinical and 2D patientspecific biomechanical data into a ML classifier. Different classification algorithms were tested through a comprehensive validation procedure in order to find the best-learned model, that turned out to be Support Vector Machine (SVM). The improvement in accuracy introduced by our model was $14 \mathrm{pp}$ over the gold-standard BMD. The results suggests that the line to follow for an effective prediction of hip fracture is the combination of both clinical and biomechanical data. Besides, this approach is economical, fast and could be integrated in the clinical routine without changing the clinical workflow.

Future research works should include a greater volume of samples, better image quality and more phenotypic attributes into the dataset. This might capture a larger part of the variance, as there are conditions, for example related to high fall risk, that can not be addressed by FEM. In addition, we did not explicitly include the fracture location into the learning procedure, because it would have meant to split the dataset, therefore having even less volume of data to learn an appropriate model. The correct identification of fracture type is a challenge for 2D finite analyses due to the inherent 2D limitation and the overlapping of cortical and trabecular bone on the image plane. With a

larger dataset, fracture location could be considered independently in order to investigate into the etiology and the best predictive attributes for each fracture type. Besides, other techniques such as meta-algorithms should be explored in the future to improve classification accuracy.

\section{Conflict of Interest}

All authors state that they have no conflicts of interest. 


\section{Acknowledgements}

This study was partially funded by two grants Cátedra UPV-Fundación Quaes, obtained by Eduardo Villamor Medina and Antonio Cutillas Pardines, and one FPI grant (FPI-SP20170111) from the Universitat Politècnica de València obtained by Eduardo Villamor Medina.

\section{References}

[1] G. Holt, R. Smith, K. Duncan, J. D. Hutchison, D. Reid, Changes in population demographics and the future incidence of hip fracture, Injury 40 (7) (2009) 722-726. doi:10.1016/j.injury.2008.11.004.

URL https://www.sciencedirect.com/science/article/pii/S0020138308005202?via\%3Dihub

[2] A. Svedbom, E. V. McCloskey, M. Ivergård, J. A. Kanis, J. Stenmark, E. Hernlund, B. Jönsson, C. Cooper, J. Compston, Osteoporosis in the European Union: medical management, epidemiology and economic burden, Archives of Osteoporosis 8 (1-2) (2013) 136. doi:10.1007/s11657-013-01361 .

URL http://link.springer.com/10.1007/s11657-013-0136-1

[3] C. Cooper, G. Campion, L. J. Melton, Hip fractures in the elderly: A world-wide projection, Osteoporosis International 2 (6) (1992) 285-289. doi:10.1007/BF01623184.

URL http://link.springer.com/10.1007/BF01623184

[4] O. Johnell, The socioeconomic burden of fractures: Today and in the 21st century, The American Journal of Medicine 103 (2) (1997) S20-S26. doi:10.1016/s0002-9343(97)90023-1.

URL https://www.sciencedirect.com/science/article/pii/S0002934397900231?via\%3Dihub

[5] C. Cooper, E. J. Atkinson, S. J. Jacobsen, W. M. O'Fallon, L. J. Melton, Population-based study of survival after osteoporotic fractures., American journal of epidemiology 137 (9) (1993) 1001-5.

URL http://www.ncbi.nlm.nih.gov/pubmed/8317445 
[6] P. Geusens, T. Van Geel, J. Van Den Bergh, Can hip fracture prediction in women be estimated beyond bone mineral density measurement alone?, Therapeutic Advances in Musculoskeletal Disease 2 (2) (2010) 63-77. doi:10.1177/1759720X09359541.

URL http://www.ncbi.nlm.nih.gov/pubmed/22870438 http://www . pubmedcentral .nih.gov/articlerender . fcgi?artid=PMC3383475

[7] S. Tomasevic-Todorovic, A. Vazic, A. Issaka, F. Hanna, Comparative assessment of fracture risk among osteoporosis and osteopenia patients: A cross-sectional study, Open Access Rheumatology: Research and Reviews 10 (2018) 61-66. doi:10.2147/OARRR.S151307.

URL http://www.ncbi.nlm.nih.gov/pubmed/29881314 http://www . pubmedcentral .nih.gov/articlerender.fcgi?artid=PMC5985792

[8] World Health Organization, Assessment of fracture risk and its application to screening for postmenopausal osteoporosis. Report of a WHO Study Group (1994).

URL http://www.who.int/iris/handle/10665/39142 http://apps.who.int//iris/handle/10665/39142

[9] A. El Maghraoui, C. Roux, DXA scanning in clinical practice, QJM: An International Journal of Medicine 101 (8) (2008) 605-617. doi:10.1093/qjmed/hen022.

URL https://academic. oup.com/qjmed/article-lookup/doi/10.1093/qjmed/hcn022 http://www.ncbi.nlm.nih.gov/pubmed/18334497

[10] T. Chevalley, R. Rizzoli, V. Nydegger, D. Slosman, L. Tkatch, C. H. Rapin, H. Vasey, J. P. Bonjour, Preferential low bone mineral density of the femoral neck in patients with a recent fracture of the proximal femur., Osteoporosis International 1 (3) (1991) 147-54.

URL http://www.ncbi.nlm.nih.gov/pubmed/1790402

[11] N. Li, X.-M. Li, L. Xu, W.-J. Sun, X.-G. Cheng, W. Tian, Clinical Study Comparison of QCT and DXA: Osteoporosis Detection Rates in 
Postmenopausal Women, International Journal of Endocrinology 2013. doi:10.1155/2013/895474.

URL http://dx.

[12] C. R. Wills, A. L. Olivares, S. Tassani, M. Ceresa, V. Zimmer, M. A. González Ballester, L. M. del Río, L. Humbert, J. Noailly, 3D patientspecific finite element models of the proximal femur based on DXA towards the classification of fracture and non-fracture cases, Bone 121 (2019) 89-99. doi:10.1016/j.bone.2019.01.001.

URL https://www.sciencedirect.com/science/article/pii/S8756328219300018?via\%3Dihub https://linkinghub.elsevier.com/retrieve/pii/S8756328219300018

[13] G. Fountoulis, T. Kerenidi, C. Kokkinis, P. Georgoulias, P. Thriskos, K. Gourgoulianis, I. Fezoulidis, K. Vassiou, M. Vlychou, Assessment of Bone Mineral Density in Male Patients with Chronic Obstructive Pulmonary Disease by DXA and Quantitative Computed Tomography., International journal of endocrinology 2016 (2016) 6169721. doi:10.1155/2016/6169721.

URL http://www.ncbi.nlm.nih.gov/pubmed/27087809 http://www . pubmedcentral .nih.gov/articlerender.fcgi?artid=PMC4819104

[14] D. Testi, M. Viceconti, F. Baruffaldi, A. Cappello, Risk of fracture in elderly patients: a new predictive index based on bone mineral density and finite element analysis, Computer Methods and Programs in Biomedicine 60 (1999) 23-33.

URL https://ac.els-cdn.com/S0169260799000073/1-s2.0-S0169260799000073-main.pdf?_tid=e58

[15] L. Yang, L. Palermo, D. M. Black, R. Eastell, Prediction of incident hip fracture with the estimated femoral strength by finite element analysis of DXA scans in the study of osteoporotic fractures, Journal of Bone and Mineral Research 29 (12) (2014) 2594-2600. doi:10.1002/jbmr.2291.

URL http://doi.wiley.com/10.1002/jbmr.2291

[16] Y. Luo, S. Ahmed, W. D. Leslie, Automation of a DXA-based finite element 
tool for clinical assessment of hip fracture risk, Computer Methods and Programs in Biomedicine 155 (2018) 75-83. doi:10.1016/j.cmpb.2017.11.020. URL https://linkinghub.elsevier.com/retrieve/pii/S0169260717310283

[17] E. Dall'Ara, R. Eastell, M. Viceconti, D. Pahr, L. Yang, Experimental validation of DXA-based finite element models for prediction of femoral strength, Journal of the Mechanical Behavior of Biomedical Materials 63 (2016) 17-25. doi:10.1016/j.jmbbm.2016.06.004.

URL https://www.sciencedirect.com/science/article/pii/S1751616116301709

[18] W. S. Enns-Bray, H. Bahaloo, I. Fleps, Y. Pauchard, E. Taghizadeh, S. Sigurdsson, T. Aspelund, P. Büchler, T. Harris, V. Gudnason, S. J. Ferguson, H. Pálsson, B. Helgason, Biofidelic finite element models for accurately classifying hip fracture in a retrospective clinical study of elderly women from the AGES Reykjavik cohort, Bone 120 (2019) 25-37. doi:10.1016/j.bone.2018.09.014.

URL https://www.sciencedirect.com/science/article/pii/S8756328218303521?via\%3Dihub

[19] M. Terzini, A. Aldieri, L. Rinaudo, G. Osella, A. L. Audenino, C. Bignardi, Improving the Hip Fracture Risk Prediction Through 2D Finite Element Models From DXA Images: Validation Against 3D Models, Frontiers in Bioengineering and Biotechnology 7 (SEP) (2019) 220. doi:10.3389/fbioe.2019.00220.

URL https://www.frontiersin.org/article/10.3389/fbioe.2019.00220/full

[20] N. D. Nguyen, S. A. Frost, J. R. Center, J. A. Eisman, T. V. Nguyen, Development of prognostic nomograms for individualizing 5-year and 10year fracture risks, Osteoporosis International 19 (10) (2008) 1431-1444. doi:10.1007/s00198-008-0588-0.

URL http://link.springer.com/10.1007/s00198-008-0588-0

[21] J. A. Kanis, A. Oden, H. Johansson, F. Borgström, O. Ström, E. McCloskey, FRAX® and its applications to clinical practice, Bone 44 (5) 
(2009) 734-743. doi:10.1016/J.BONE.2009.01.373.

URL https://www.sciencedirect.com/science/article/pii/S8756328209004049?via\%3Dihub

[22] M. J. Bolland, A. T. Siu, B. H. Mason, A. M. Horne, R. W. Ames, A. B. Grey, G. D. Gamble, I. R. Reid, Evaluation of the FRAX and Garvan fracture risk calculators in older women, Journal of Bone and Mineral Research 26 (2) (2011) 420-427. doi:10.1002/jbmr.215.

URL http://doi.wiley.com/10.1002/jbmr.215

[23] T. P. Ho-Le, J. R. Center, J. A. Eisman, T. V. Nguyen, H. T. Nguyen, Prediction of hip fracture in post-menopausal women using artificial neural network approach, in: Proceedings of the Annual International Conference of the IEEE Engineering in Medicine and Biology Society, EMBS, IEEE, 2017, pp. 4207-4210. doi:10.1109/EMBC.2017.8037784.

URL https://ieeexplore.iee.org/document/8037784/

[24] C. Kruse, P. Eiken, P. Vestergaard, Clinical fracture risk evaluated by hierarchical agglomerative clustering, Osteoporosis International 28 (3) (2017) 819-832. doi:10.1007/s00198-016-3828-8.

URL http://link.springer.com/10.1007/s00198-016-3828-8

[25] P. K. Saha, U. Ferizi, H. Besser, C. Chen, C. S. Rajapakse, J. Jacobs, P. Hysi, G. Chang, S. Honig, Artificial Intelligence Applied to Osteoporosis: A Performance Comparison of Machine Learning Algorithms in Predicting Fragility Fractures From MRI Data, Journal of Magnetic Resonance Imagingdoi:10.1002/jmri.26280.

URL http://www.ncbi.nlm.nih.gov/pubmed/30252971

http://doi.wiley.com/10.1002/jmri. 26280

[26] K. K. Nishiyama, M. Ito, A. Harada, S. K. Boyd, Classification of women with and without hip fracture based on quantitative computed tomography and finite element analysis, Osteoporosis International 25 (2) (2014) 619-626. doi:10.1007/s00198-013-2459-6.

URL https://link. springer. com/content/pdf/10.1007\%2Fs00198-013-2459-6.pdf 
[27] P. Jiang, S. Missoum, Z. Chen, Fusion of clinical and stochastic finite element data for hip fracture risk prediction, Journal of Biomechanics 48 (15) (2015) 4043-4052. doi:10.1016/j.jbiomech.2015.09.044.

URL https://www.sciencedirect.com/science/article/pii/S0021929015005400?via\%3Dihub

[28] K. E. Naylor, E. V. McCloskey, R. Eastell, L. Yang, Use of DXA-based finite element analysis of the proximal femur in a longitudinal study of hip fracture, Journal of Bone and Mineral Research 28 (5) (2013) 1014-1021. doi:10.1002/jbmr.1856.

URL http://doi.wiley.com/10.1002/jbmr.1856

[29] S. A. Maas, B. J. Ellis, G. A. Ateshian, J. A. Weiss, FEBio: Finite Elements for Biomechanics, Journal of Biomechanical Engineering 134 (1) (2012) 011005. doi:10.1115/1.4005694.

URL http://biomechanical . asmedigitalcollection . asme.org/article.aspx?articleid=1431396

[30] A. E. H. Love, A treatise on the mathematical theory of elasticity, Dover Publications, 1944.

URL https://books.google.es/books/about/A_Treatise_on_the_Mathematical_Theory_of .html?id=

[31] T. Rossman, V. Kushvaha, D. Dragomir-Daescu, QCT/FEA predictions of femoral stiffness are strongly affected by boundary condition modeling, Computer Methods in Biomechanics and Biomedical Engineering 19 (2) (2016) 208-216. doi:10.1080/10255842.2015.1006209.

URL http://www.ncbi.nlm.nih.gov/pubmed/25804260 http://www.pubmedcentral.nih.gov/articlerender.fcgi?artid=PMC4583333 http://www.tandfonline.com/doi/full/10.1080/10255842.2015.1006209

[32] H. Si, TetGen, a Delaunay-Based Quality Tetrahedral Mesh Generator, ACM Transactions on Mathematical Software 41 (2) (2015) 1-36. doi:10.1145/2629697.

URL http://dl.acm.org/citation.cfm?doid=2732672.2629697

[33] L. Yang, N. Peel, J. A. Clowes, E. V. McCloskey, R. Eastell, Use of DXAbased structural engineering models of the proximal femur to discriminate 
hip fracture, Journal of Bone and Mineral Research 24 (1) (2009) 33-42. doi:10.1359/jbmr.080906.

URL http://doi.wiley.com/10.1359/jbmr.080906

[34] E. Schileo, E. Dall'Ara, F. Taddei, A. Malandrino, T. Schotkamp, M. Baleani, M. Viceconti, An accurate estimation of bone density improves the accuracy of subject-specific finite element models, Journal of Biomechanics 41 (11) (2008) 2483-2491. doi:10.1016/j.jbiomech.2008.05.017.

URL http://www.ncbi.nlm.nih.gov/pubmed/18606417 https://linkinghub.elsevier.com/retrieve/pii/S0021929008002467

[35] E. F. Morgan, T. M. Keaveny, Dependence of yield strain of human trabecular bone on anatomic site, Journal of Biomechanics 34 (5) (2001) 569-577. doi:10.1016/S0021-9290(01)00011-2.

URL https://www.sciencedirect.com/science/article/pii/S0021929001000112?via\%3Dihub

[36] E. F. Morgan, H. H. Bayraktar, T. M. Keaveny, Trabecular bone modulus-density relationships depend on anatomic site, Journal of Biomechanics 36 (7) (2003) 897-904. doi:10.1016/S0021-9290(03)00071-X.

URL https://www.sciencedirect.com/science/article/pii/S002192900300071X?via\%3Dihub

[37] H. H. Bayraktar, E. F. Morgan, G. L. Niebur, G. E. Morris, E. K. Wong, T. M. Keaveny, Comparison of the elastic and yield properties of human femoral trabecular and cortical bone tissue, Journal of Biomechanics 37 (1) (2004) 27-35. doi:10.1016/S0021-9290(03)00257-4.

URL https://www.sciencedirect.com/science/article/pii/S0021929003002574?via\%3Dihub

[38] K. Ün, G. Bevill, T. M. Keaveny, The effects of side-artifacts on the elastic modulus of trabecular bone, Journal of Biomechanics 39 (11) (2006) 1955-1963. doi:10.1016/J.JBIOMECH.2006.05.012.

URL https://www.sciencedirect.com/science/article/pii/S0021929006001667?via\%3Dihub

[39] E. Schileo, L. Balistreri, L. Grassi, L. Cristofolini, F. Taddei, To what extent can linear finite element models of human femora predict failure 
under stance and fall loading configurations?, Journal of Biomechanics 47 (14) (2014) 3531-3538. doi:10.1016/J.JBIOMECH.2014.08.024.

URL https://www.sciencedirect.com/science/article/pii/S0021929014004606?via\%3Dihub

[40] D. C. Wirtz, N. Schiffers, T. Pandorf, K. Radermacher, D. Weichert, R. Forst, Critical evaluation of known bone material properties to realize anisotropic FE-simulation of the proximal femur, Journal of Biomechanics 33 (10) (2000) 1325-1330. doi:10.1016/S0021-9290(00)00069-5.

URL https://www.sciencedirect.com/science/article/pii/S0021929000000695?via\%3Dihub

[41] F. Eckstein, C. Wunderer, H. Boehm, V. Kuhn, M. Priemel, T. M. Link, E.-M. Lochmüller, Reproducibility and Side Differences of Mechanical Tests for Determining the Structural Strength of the Proximal Femur, Journal of Bone and Mineral Research 19 (3) (2003) 379-385. doi:10.1359/JBMR.0301247.

URL http://doi.wiley.com/10.1359/JBMR.0301247

[42] E. S. Orwoll, L. M. Marshall, C. M. Nielson, S. R. Cummings, J. Lapidus, J. A. Cauley, K. Ensrud, N. Lane, P. R. Hoffmann, D. L. Kopperdahl, T. M. Keaveny, Osteoporotic Fractures in Men Study Group, Finite element analysis of the proximal femur and hip fracture risk in older men., Journal of bone and mineral research : the official journal of the American Society for Bone and Mineral Research 24 (3) (2009) 475-83. doi:10.1359/jbmr.081201.

URL http://www.ncbi.nlm.nih.gov/pubmed/19049327

http://www . pubmedcentral.nih.gov/articlerender.fcgi?artid=PMC2659519

[43] S. Abe, A. Ylinen, N. Narragirish, R. Nikander, J. Hyttinen, R. Kouhia, H. Sievänen, Exploration of different boundary conditions in the sideways falling situation in hip fracture finite element modeling, in: Proceedings of the XII Finnish Mechanics Days, 2015, pp. 130-135.

URL http://meshlab.sourceforge.net/

[44] W. J. Choi, P. A. Cripton, S. N. Robinovitch, Effects of hip abductor 
muscle forces and knee boundary conditions on femoral neck stresses during simulated falls, Osteoporosis International 26 (1) (2015) 291-301. doi:10.1007/s00198-014-2812-4.

URL http://www.ncbi.nlm.nih.gov/pubmed/25027112

http://link. springer . com/10 .1007/s00198-014-2812-4

[45] A. J. van den Kroonenberg, W. C. Hayes, T. A. McMahon, Dynamic Models for Sideways Falls From Standing Height, Journal of Biomechanical Engineering 117 (3) (1995) 309. doi:10.1115/1.2794186.

URL http://biomechanical . asmedigitalcollection . asme.org/article . aspx?articleid=1400177

[46] S. N. Robinovitch, W. C. Hayes, T. A. McMahon, Prediction of Femoral Impact Forces in Falls on the Hip, Journal of Biomechanical Engineering 113 (4) (1991) 366. doi:10.1115/1.2895414.

URL http://www.ncbi.nlm.nih.gov/pubmed/1762432 http://biomechanical . asmedigitalcollection. asme.org/article. aspx?articleid=1398671

[47] S. N. Robinovitch, T. A. McMahon, W. C. Hayes, Force attenuation in trochanteric soft tissues during impact from a fall, Journal of Orthopaedic Research 13 (6) (1995) 956-962. doi:10.1002/jor.1100130621.

URL http://doi.wiley.com/10.1002/jor.1100130621

[48] A. B. Dufour, B. Roberts, K. E. Broe, D. P. Kiel, M. L. Bouxsein, M. T. Hannan, The factor-of-risk biomechanical approach predicts hip fracture in men and women: the Framingham Study., Osteoporosis International 23 (2) (2012) 513-20. doi:10.1007/s00198-011-1569-2.

URL http://www.ncbi.nlm.nih.gov/pubmed/21344243 http://www . pubmedcentral.nih.gov/articlerender . fcgi?artid=PMC3289518

[49] E. Schileo, F. Taddei, L. Cristofolini, M. Viceconti, Subject-specific finite element models implementing a maximum principal strain criterion are able to estimate failure risk and fracture location on human femurs tested in vitro, Journal of Biomechanics 41 (2) (2008) 356-367. 
doi:10.1016/J.JBIOMECH.2007.09.009.

URL https://www.sciencedirect.com/science/article/pii/S0021929007003752

[50] C. Mautalen, E. Vega, T. Einhorn, Are the etiologies of cervical and trochanteric hip fractures different?, Bone 18 (3) (1996) S133-S137. doi:10.1016/8756-3282(95)00490-4.

URL https://www.sciencedirect.com/science/article/pii/8756328295004904

[51] S. Yang, W. D. Leslie, Y. Luo, A. L. Goertzen, S. Ahmed, L. M. Ward, I. Delubac, L. M. Lix, Automated DXA-based finite element analysis for hip fracture risk stratification: a cross-sectional study, Osteoporosis International 29 (1) (2018) 191-200. doi:10.1007/s00198-017-4232-8.

URL http://link.springer.com/10.1007/s00198-017-4232-8

[52] D. Testi, M. Viceconti, A. Cappello, S. Gnudi, Prediction of Hip Fracture Can Be Significantly Improved by a Single Biomedical Indicator, Annals of Biomedical Engineering 30 (6) (2002) 801-807. doi:10.1114/1.1495866.

URL http://link.springer.com/10.1114/1.1495866

[53] C. M. Langton, S. Pisharody, J. H. Keyak, Generation of a 3D proximal femur shape from a single projection 2D radiographic image, Osteoporosis International 20 (3) (2009) 455-461. doi:10.1007/s00198-008-0665-4.

URL http://link.springer.com/10.1007/s00198-008-0665-4

[54] L. Humbert, Y. Martelli, R. Fonolla, M. Steghofer, S. Di Gregorio, J. Malouf, J. Romera, L. M. D. R. Barquero, 3D-DXA: Assessing the Femoral Shape, the Trabecular Macrostructure and the Cortex in 3D from DXA images, IEEE Transactions on Medical Imaging 36 (1) (2017) 27-39. doi:10.1109/TMI.2016.2593346.

URL http://ieeexplore.ieee.org/document/7516699/

[55] J. H. Keyak, S. Sigurdsson, G. Karlsdottir, D. Oskarsdottir, A. Sigmarsdottir, S. Zhao, J. Kornak, T. B. Harris, G. Sigurdsson, B. Y. Jonsson, K. Siggeirsdottir, G. Eiriksdottir, V. Gudnason, T. F. Lang, Male-female 
differences in the association between incident hip fracture and proximal femoral strength: a finite element analysis study., Bone 48 (6) (2011) 1239-45. doi:10.1016/j.bone.2011.03.682.

URL http://www.ncbi.nlm.nih.gov/pubmed/21419886 http://www . pubmedcentral . nih.gov/articlerender . fcgi?artid=PMC3095704

[56] E. Lobo, G. Marcos, J. Santabárbara, H. Salvador-Rosés, L. Lobo-Escolar, C. D. la Cámara, A. Aso, A. Lobo-Escolar, Gender differences in the incidence of and risk factors for hip fracture: A 16-year longitudinal study in a southern European population, Maturitas 97 (2017) 38-43. doi:10.1016/J.MATURITAS.2016.12.009.

URL https://www .maturitas .org/article/S0378-5122(16)30454-6/fulltext 